\title{
Comparison of acute myeloid leukemia and myelodysplastic syndromes with TP53 aberrations
}

\author{
Sayantanee Dutta ${ }^{1}$ Jennifer Moritz ${ }^{1}$. Gudrun Pregartner ${ }^{2}$ - Gerhard G. Thallinger ${ }^{3,4}$ • Ilona Brandstätter ${ }^{1}$. \\ Karin Lind ${ }^{1} \cdot \operatorname{Simin}$ Rezania $^{1} \cdot$ Freya Lyssy ${ }^{4}$ - Andreas Reinisch ${ }^{1,5} \cdot$ Armin Zebisch $^{1,6} \cdot$ Andrea Berghold $^{2}$. \\ Albert Wölfler ${ }^{1} \cdot$ Heinz Sill ${ }^{1,4}$ (i)
}

Received: 26 November 2021 / Accepted: 13 January 2022 / Published online: 26 January 2022

(c) The Author(s) 2022

\begin{abstract}
TP53 aberrations are found in approximately $10 \%$ of patients with acute myeloid leukemia (AML) and myelodysplastic syndromes (MDS) and are considered early driver events affecting leukemia stem cells. In this study, we compared features of a total of 84 patients with these disorders seen at a tertiary cancer center. Clinical and cytogenetic characteristics as well as immunophenotypes of immature blast cells were similar between AML and MDS patients. Median overall survival (OS) was 226 days (95\% confidence interval [CI], 131-300) for the entire cohort with an estimated 3-year OS rate of $11 \%$ (95\% CI, 6-22). OS showed a significant difference between MDS (median, 345 days; 95\% CI, 235-590) and AML patients (median, 91 days; 95\% CI, 64-226) which is likely due to a different co-mutational pattern as revealed by next-generation sequencing. Transformation of TP53 aberrant MDS occurred in $60.5 \%$ of cases and substantially reduced their survival probability. Cox regression analysis revealed treatment class and TP53 variant allele frequency as prognostically relevant parameters but not the TP53-specific prognostic scores EAp53 and RFS. These data emphasize similarities between TP53 aberrant AML and MDS and support previous notions that they should be classified and treated as a distinct disorder.
\end{abstract}

Keywords TP53 · Acute myeloid leukemia $\cdot$ Myelodysplastic syndromes $\cdot$ Stem cell disorder $\cdot$ EAp53 and RFS score

\section{Introduction}

The TP53 gene, located at chromosome 17p13.1, encodes p53-a fundamental tumor suppressor highly conserved during evolution. Among a multitude of different physiological

Heinz Sill

heinz.sill@medunigraz.at

1 Division of Hematology, Medical University of Graz, Auenbruggerplatz 38, 8036 Graz, Austria

2 Institute for Medical Informatics, Statistics and Documentation, Medical University of Graz, Graz, Austria

3 Institute of Biomedical Informatics, Graz University of Technology, Graz, Austria

4 BioTechMed-Graz, Graz, Austria

5 Department of Blood Group Serology and Transfusion Medicine, Medical University of Graz, Graz, Austria

6 Otto-Loewi-Research Center for Vascular Biology, Immunology and Inflammation, Division of Pharmacology, Medical University of Graz, Graz, Austria functions, p53 is activated by several extrinsic and intrinsic stress signals including DNA damage, oncogene activation, hypoxia, and nutrient deprivation. Dependent on the activation signal, p53 induces a multitude of downstream signals aimed at sustaining cellular homeostasis. Importantly, by pursuing these functions, p53 acts in a cell context-specific manner. There is tight regulation of p53 at the transcriptional, post-transcriptional, and post-translational levels, respectively, conferring fine-tuning of this essential cellular protein $[1,2]$.

p53 displays pivotal functions in normal hematopoietic stem and progenitor cells being involved in their proliferation, differentiation, and apoptosis [3]. Using transgenic mice, it could be shown that 533 conveys quiescence during steady-state hematopoiesis [4]. Murine p53-deficient stem cells showed enhanced self-renewal with increased serial replating and repopulating capacity in vitro and in vivo, respectively. In cooperation with oncogenic mutations like Kras ${ }^{\mathrm{G} 12 \mathrm{D}}$, p53 loss led to indefinite self-renewal of these cells with a propensity for transformation into leukemiainitiating cells [5]. 
Acute myeloid leukemia (AML) is an aggressive hematopoietic malignancy derived from transformed HSPCs ultimately leading to bone marrow failure. Myelodysplastic syndromes (MDS) are clonal bone marrow disorders characterized by ineffective hematopoiesis and peripheral blood cytopenias progressing to AML in a substantial number of cases. Both disease entities are highly heterogeneous with respect to biological and clinical features [6, 7]. In AML and MDS, TP53 aberrations are constantly observed at a frequency of approximately $10 \%$ with steep increases in older patients and those with therapy-related myeloid neoplasms. Predominantly, they constitute missense mutations located in the DNA binding domain of the gene. However, other types of mutations-nonsense variants, small insertions and deletions-as well as chromosomal losses encompassing the TP53 locus and combined aberrations are also observed $[8,9]$. These aberrations may lead to loss of physiological functions, exert a dominant-negative phenotype on wild-type p53, or - in some instances - result in a gain of novel, oncogenic properties. Concerning their origin, TP53 mutations are somatically acquired in the majority of patients affecting hematopoietic stem and progenitor cells; however, germline mutations characterizing the Li-Fraumeni (LF) and LF-like syndromes are also observed in these disorders [10-15]. Recently, it was reported that individuals with clonal hematopoiesis of indeterminate potential with TP53 mutations exhibit a high risk of progression to AML [16]. Patients with AML and MDS with TP53 aberrations usually have a dismal prognosis even when treated with intensive regimens including allogeneic hematopoietic stem cell transplantation (HSCT) [17, 18].

Based on biological and clinical features, we recently proposed that AML and MDS with TP53 aberrations should be regarded as a distinct disease entity [19]. In this work, we compare data from such patient cohorts referred to a tertiary hematology center to corroborate our proposition.

\section{Methods}

The study cohort consisted of adult patients suffering from AML and MDS with TP53 aberrations, seen at our institution-a tertiary hematology center for a population of approximately 1.5 million people—between November 2014 and June 2021. Only those patients whose TP53 aberrations were detected at diagnosis were included. Diagnosis and risk stratification of myeloid neoplasms were performed according to standard criteria including conventional karyotyping as well as fluorescent in situ hybridization (FISH). For molecular studies, genomic DNA extracted from bone marrow biopsies was analyzed for mutations in up to 44 myeloid-associated genes using an Ion Torrent next-generation sequencing (NGS) platform (Thermo Fisher Scientific) as described previously [20]. With respect to TP53, all coding exons and flanking exon-intron boundaries were sequenced with a lower limit of detection set at $5 \%$ mutant allele reads. Variants were classified according to the VarSome (https:// varsome.com) and TP53 databases (https://p53.iacr.fr), and only pathogenic and likely pathogenic ones were included. Treatment categories comprised intensive chemotherapy and allogeneic hematopoietic stem cell transplantation (HSCT), non-intensive therapies (lenalidomide, hypomethylating agents, low-dose cytarabine, and growth factors), and best supportive care including hydroxyurea. All patient data were retrieved from openMEDOCS, a regional hospital-based documentation system.

\section{Statistical analysis}

To compare clinical and biological parameters, the Mann-Whitney $U$ and Fisher's exact tests were used for continuous and categorical parameters, respectively, and median (range) or numbers ( $n$, in \%) are used to descriptively summarize the data. Kaplan-Meier analysis and the log-rank test were used to describe and compare overall survival (OS), calculated as time from diagnosis to either death or last-follow up, and the median OS with $95 \%$ confidence intervals (CI) is presented. To identify relevant prognostic parameters, Cox regression analyses were performed taking the particular disease (AML, MDS), age, sex, the TP53 variant allele frequency (VAF), TP53-specific scores, and the treatment class into account. The TP53 scores were explored as previously described by our group [21]. Briefly, we initially assessed the impact of the location and consecutive amino acid alteration of a particular TP53 variant. Then, we investigated the evolutionary action score (EAp53) focusing on the evolutionary sensitivity to sequence variation and amino acid conservation of missense mutations. Finally, the relative fitness score (RFS) based on in vitro growth properties of particular TP53 variants was evaluated. For the multivariable analysis, all parameters with $p<0.05$ in the univariable analysis as well as age and sex were considered. The analyses were performed using R version 4.1.0 (https:// www.R-project.org/).

\section{Immunophenotyping}

The immunophenotype of blast cells was compared between AML and MDS patients showing TP53 aberrations. Multicolor flow cytometry was performed using a Navios cytometer (Beckman Coulter, USA) with harmonized baseline settings as described previously [22]. After erythrocyte lysis, peripheral blood $(\mathrm{PB})$ or bone marrow $(\mathrm{BM})$ cells were washed twice with D-PBS (Life Technologies) and stained with the appropriate antibodies (Supplementary Table 1) for 18 min in the dark. At least 30,000 events were recorded and 
data were analyzed using Kaluza software (Beckman Coulter). Blasts were defined by $\mathrm{CD} 45^{\mathrm{dim}} / \mathrm{SSC}^{\text {low }}$ gating with additional backgating using $\mathrm{CD} 34^{-}$and $\mathrm{CD} 117^{-}$expression to improve the identification of the blast population when appropriate. For all markers, isotype controls were used to define the percentage of marker positive cells. In addition, the mean fluorescence intensity (MFI) ratio was calculated by dividing the geometric MFI of the antibody-stained sample by the geometric MFI of the respective isotype control. MFI ratio values $>1.5$ were considered as significant [23]. MFI ratios as well as percent positive cells (PPCs) for each marker were compared between TP53 aberrant AML and MDS samples using the Mann-Whitney $U$ test. Multiple testing correction was performed using the Benjamini-Hochberg procedure to control the false discovery rate [24]. A $p$ value below 0.05 was considered statistically significant. Principal component analysis (PCA) was performed on scaled MFI ratio and PPC data and visualized as a biplot of principal components 1 and 2 , respectively, using the $\mathrm{R}$ package ggbiplot (v. 0.55) (https://github.com/vqv/ggbiplot). Samples with missing values were removed prior to PCA. Statistical analysis was performed with GraphPad Prism software version 9.1 (GraphPad Software) and R (v. 4.1.2).

\section{Results}

A total of 84 patients with a median age of 71 years (range 34-86) suffering from myeloid malignancies with TP53 aberrations were included, 46 of them with AML and 38 with MDS. The median observation period for the total cohort was 149 days (range, 5-2947). Demographic data of the patients are depicted in Table 1. Notably, de novo AML was the most frequent subtype in this disease category (60.8\%), whereas therapy-related MDS accounted for the majority of MDS cases (42.0\%). Transformation of MDS to AML was observed in 23 patients $(60.5 \%)$ at a median time of 138 days (range, 30-2200) post diagnosis. With respect to laboratory parameters, platelet counts and LDH levels showed statistically significant differences between AML and MDS ( $p=0.004$ and $p=0.005$, respectively, Mann-Whitney $U$ test). Intensive chemotherapy treatments (" $3+7$ " regimen) were more frequently given to AML patients, and allogeneic HSCT was performed in 5 AML (10.9\%) and 3 MDS (8.1\%) patients, respectively. In MDS patients, non-intensive therapies, preferably the hypomethylating drug azacitidine, were most commonly applied.
Table 1 Demographic data of study patients. Values are median (range) for continuous data or numbers $(n$, in $\%)$ for categorical data. $t A M L$ and $t M D S$, therapy-related AML and MDS; $s A M L$, secondary AML; $M P N$, myeloproliferative neoplasm; $M L D$, multilineage dysplasia; $\operatorname{del}(5 q)$, deletion of $5 \mathrm{q} ; W B C$, white blood cells

\begin{tabular}{lllr}
\hline Characteristics & AML, $n=46$ & MDS, $n=38$ & $p$ value \\
\hline Age (years) & $70(34-84)$ & $72(41-86)$ & 0.242 \\
Sex & $24(52.2 \%)$ & $17(44.7 \%)$ & 0.519 \\
Female & & & \\
Disease classification & & & \\
AML & $28(60.8 \%)$ & & \\
De novo & $14(30.4 \%)$ & & \\
tAML & $4(8.6 \%)$ & $15(39.3 \%)$ & \\
sAML post MPN & & $5(13.1 \%)$ & \\
MDS & & $2(5.2 \%)$ & \\
MDS-EB & & $16(42.0 \%)$ & \\
MDS with MLD & & $23(60.5 \%)$ & \\
MDS with isolated del(5q) & & $17(44.7 \%)$ & 0.015 \\
tMDS & $17(36.9 \%)$ & & \\
Transformation to AML & & $2.9(1.1-23.5)$ & \\
Prior malignancy & $10(21.7 \%)$ & $10(0-19)$ & 0.728 \\
Laboratory values & $2.9(0.8-123.7)$ & $85.5(14-633)$ & \\
WBC (G/L) & $55(20-90)$ & $8.9(6.9-12.6)$ & \\
Bone marrow blasts (\%) & $44(9-306)$ & $52(127-1412)$ & \\
Platelets (G/L) & $8.8(5.4-12.9)$ & & \\
Hemoglobin (g/dL) & $373(114-1052)$ & & \\
Lactate dehydrogenase (U/L) & & & \\
Treatment & & & \\
Intensive chemotherapy & & & \\
Non-intensive therapy & & & \\
Best supportive care & & & \\
\hline
\end{tabular}


Cytogenetic and molecular genetic data including TP53specific risk scores are shown in Table 2, Fig. 1, and Supplementary Table 2. Rates of complex karyotypes and deletions at $17 \mathrm{p} 13.1$, the locus of the TP53 gene, were similar in both disease entities. Also, the number of patients with multiple
TP53 variants and the median TP53 VAF was comparable between AML and MDS. The number of cooperating gene mutations as revealed by NGS of a panel of myeloid-associated genes was low in both groups. However, the genes affected differed between both groups with mutations in
Table 2 Genetic characteristics of diagnostic AML and MDS samples. Cytogenetic data are based on conventional karyograms and fluorescence in situ hybridization analyses. Values are median (range) for continuous data or numbers $(n$, in $\%$ ) for categorical data. $V A F$, variant allele frequency

\begin{tabular}{llll}
\hline Characteristics & AML & MDS & $p$ value \\
\hline Cytogenetics & & & \\
$\quad$ Normal karyotype & $3 / 40(7.5 \%)$ & $2 / 28(7.1 \%)$ & 1.000 \\
Complex karyotype & $36 / 40(90.0 \%)$ & $20 / 28(71.4 \%)$ & 0.060 \\
$\quad$ Monosomy 17, deletion 17p13.1 & $19 / 40(47.5 \%)$ & $11 / 28(39.3 \%)$ & 0.621 \\
Molecular genetics & & & \\
Patients with > 1 TP53 variant & $8 / 46(17.4 \%)$ & $10 / 38(26.3 \%)$ & 0.424 \\
TP53 VAF (\%) & $60(8.9-98)$ & $53.2(4-91)$ & 0.643 \\
Availability of NGS data & $31 / 46(67.4 \%)$ & $25 / 38(65.8 \%)$ & \\
Patients with co-occurring mutations & $21 / 32(65.6 \%)$ & $17 / 25(68.0 \%)$ & 1.000 \\
No. of co-occurring mutations & $1(1-3)$ & $2(1-6)$ & 0.379 \\
\hline
\end{tabular}

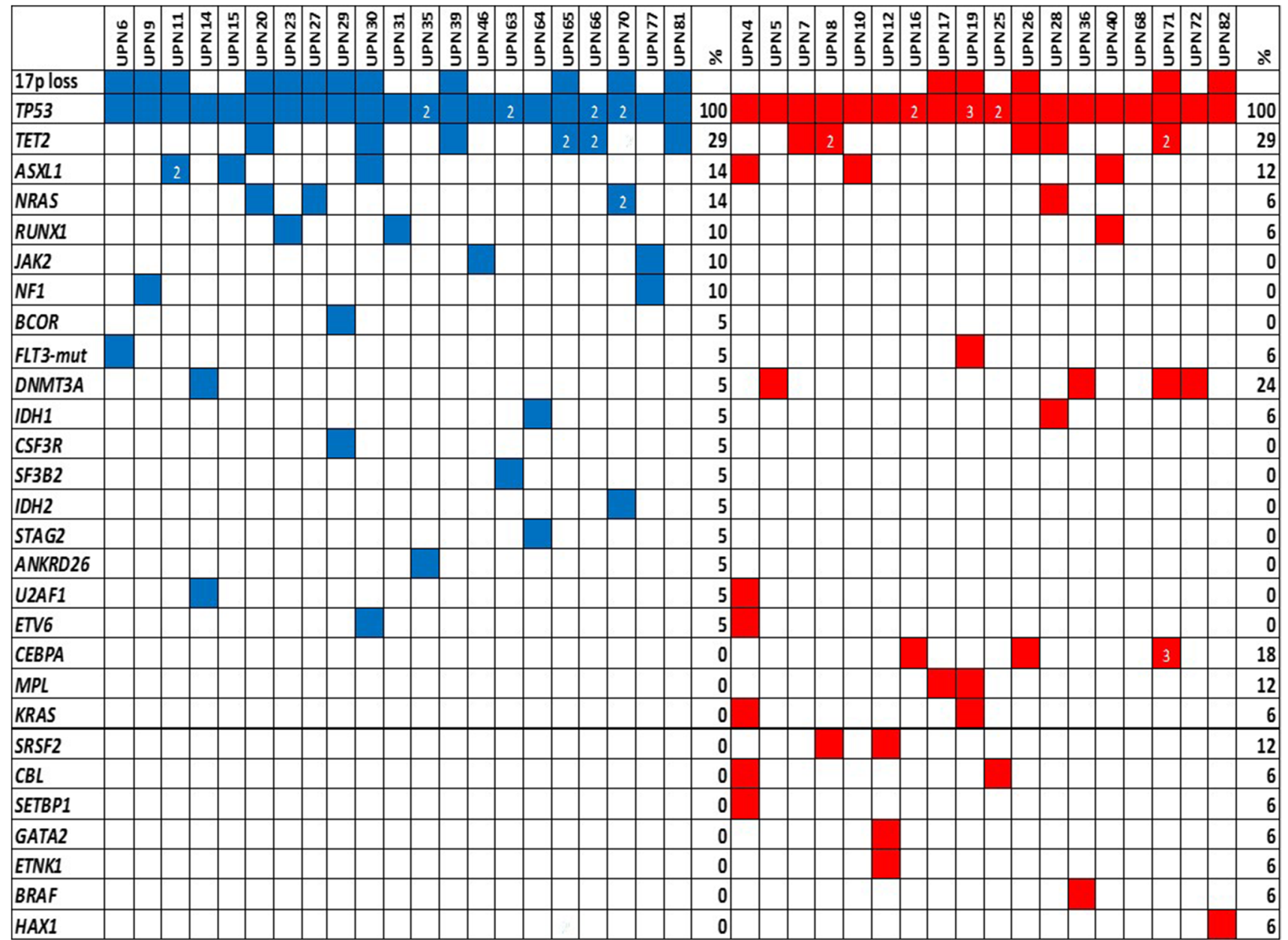

Fig. 1 Cooperative mutations detected in patients with AML (blue) and MDS (red) with TP53 aberrations. UPN, unique patient number; \% refers to patients with a particular variant; numbers within boxes indicate multiple mutations within that gene 
$K R A S, S R S F 2, D N M T 3 A$, and CEBPA being more common in MDS. Due to the small number of cases, we refrained from statistical evaluation here.

Immunophenotyping data obtained by multiparameter flow cytometry analysis at diagnosis were available in 26 AML and 18 MDS patients, respectively. By gating immature blast cells, we did not observe differences in the PPCs expressing typical markers of myeloid progenitor cells, such as CD13, CD33, CD117, CD123, and HLA-DR between diagnostic AML and MDS samples (Fig. 2A). Accordingly, expression levels of these markers as determined by MFI ratios did not differ, either (Supplementary Fig. 1). When analyzing aberrant marker expression on immature blast cells, we found significantly higher PPCs expressing CD7 in MDS as compared to AML samples. Other recurrently expressed aberrant markers, such as CD2, CD4, CD5, CD11b, CD14, CD15, CD19, and CD56, however, did not differ (Fig. 2B). Likewise, expression levels of aberrant markers were comparable in AML and MDS samples
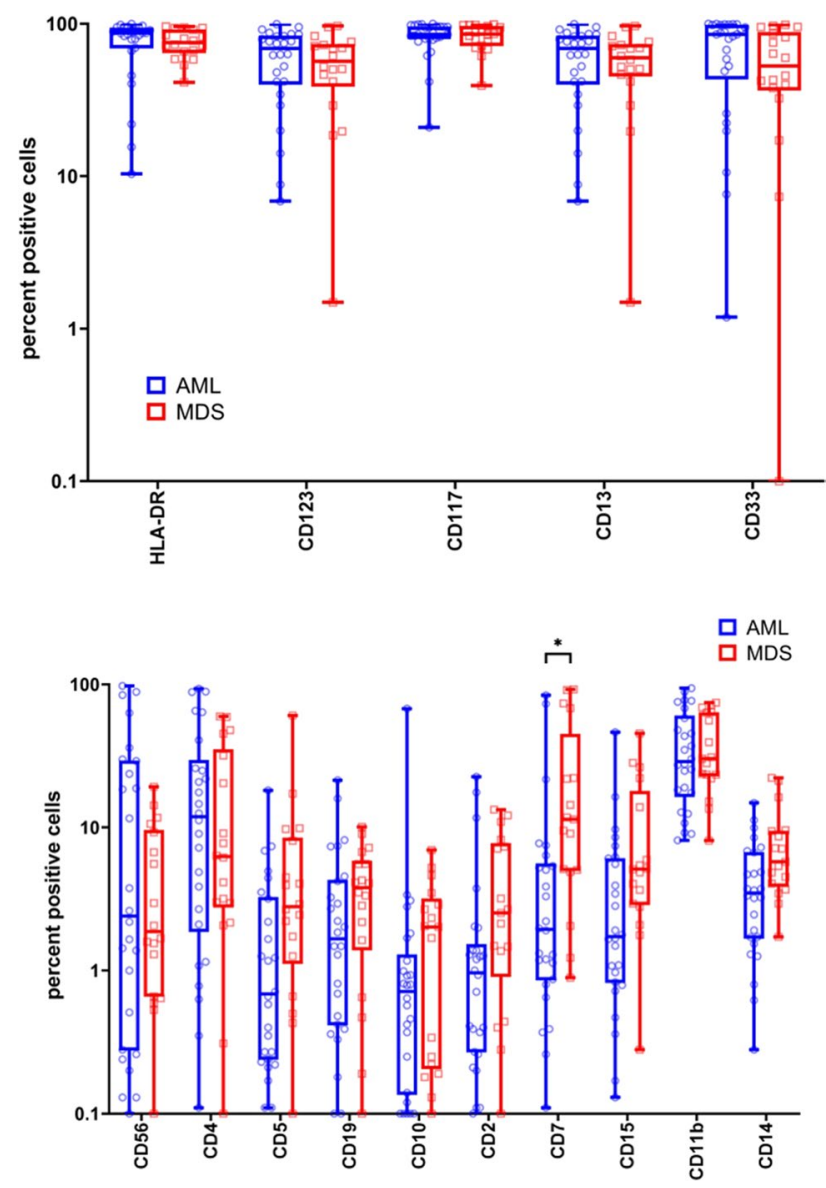

Fig. 2 Percent positive cells expressing various aberrant (A) or myeloid progenitor markers $(\mathbf{B})$ in AML $(n=26)$ and MDS samples $(n=18)$ as determined by flow cytometry analysis. Statistically significant differences in PPCs were only noted for CD7. ${ }^{*} p<0.05$ as determined by the Mann-Whitney $U$ test adjusted for multiple testing
(Supplementary Fig. 2). In five patients, paired material at MDS diagnosis and AML progression was available. Myeloid as well as aberrant marker expression varied markedly between cases but were stable between disease stages in the individual subject (Supplementary Figs. 3 and 4). Finally, PCA of PPCs as well as of MFI ratios revealed that TP53 aberrant AML and MDS samples could not be separated by immunophenotyping (Fig. 3).

The median OS of the entire cohort was 226 days $(95 \%$ CI, 131-300) (Fig. 4A) and was identical when patients were censored at allogeneic HSCT (95\% CI, 131-298). Patients with MDS had a significantly better OS with a median of 345 days (95\% CI, 235-590) as compared to those with AML with a median of 91 days (95\% CI, 64-226) (Fig. 4B). Transformation of MDS to AML substantially reduced the prognosis of these patients (Fig. 4C). When calculated from the time of MDS diagnosis, the transformed group showed a median OS of 326 days (95\% CI, 215-685), whereas the non-transformed group had one of 522 days $(95 \% \mathrm{CI}$, 227-not reached). However, when calculated from the time of transformation, AML and transformed MDS patients had a similar median OS (Supplementary Fig. 5). The estimated 3-year OS rate was $11 \%$ for all patients (95\% CI, 6-22), $7 \%$ for patients with AML $(95 \% \mathrm{CI}, 2-25)$, and $16 \%$ for those with MDS (95\% CI, 7-36), respectively. With respect to treatment classes, patients with MDS showed superior survival as compared to those with AML throughout: intensive treatment including allogeneic HSCT, 1967.5 versus 255 days $(p=0.073)$; non-intensive treatment, 463 versus 98 days $(p<0.001)$; and best supportive care, 187 versus 32.5 days $(p<0.001)$.

Table 3 presents the results of univariable and multivariable Cox regression analyses assessing the impact of conventional risk parameters, the TP53 VAF as well as the TP53specific scores. In both uni- and multivariable analyses, the presence of AML and best supportive care as treatment as well as higher TP53 VAFs were associated with a significantly shorter OS. However, neither of the TP53-specific risk scores reached statistical significance and were therefore not considered in the multivariable analysis.

\section{Discussion}

Here, we present data comparing patients with TP53 aberrant AML and MDS evaluated and treated at a tertiary cancer center. In agreement with our recently published postulation that they constitute a distinct disease entity [19], a multitude of features were, indeed, similar including clinical and cytogenetic parameters. In addition, the immunophenotype of immature blast cells did not differ significantly and a PCA revealed that TP53 aberrant AML and MDS are undistinguishable by this approach. With a median OS of 

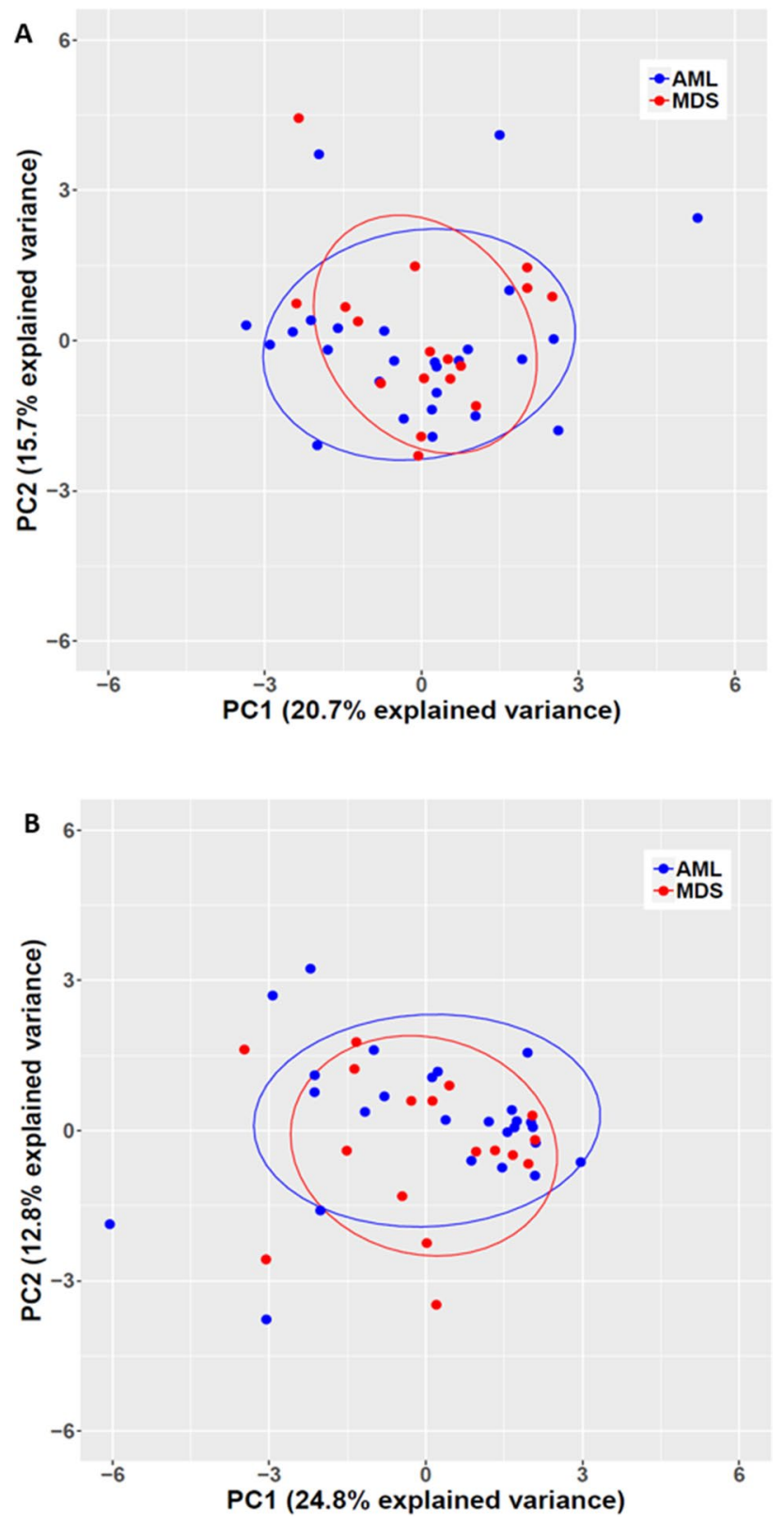

Fig. 3 Principal component analysis (PCA) of immunophenotypic marker expression on immature blast cells of TP53 aberrant AML (blue) and MDS (red) patients. A PCA of percent marker positive cells was used to evaluate the delineation of patients based on FACS analysis of 15 cell surface markers. AML and MDS samples cannot be separated by principal components 1 and 2, which explain $20.7 \%$ and $15.7 \%$ of the total variance, respectively. B PCA of the MFI ratio was used to evaluate the delineation of patients based on FACS analysis of 16 cell surface markers. AML and MDS samples cannot be separated by principal components 1 and 2, which explain $24.8 \%$ and $12.8 \%$ of the total variance, respectively. The points reflect the scores of the subjects; samples of a group are enclosed by a concentration ellipse with $68 \%$ probability
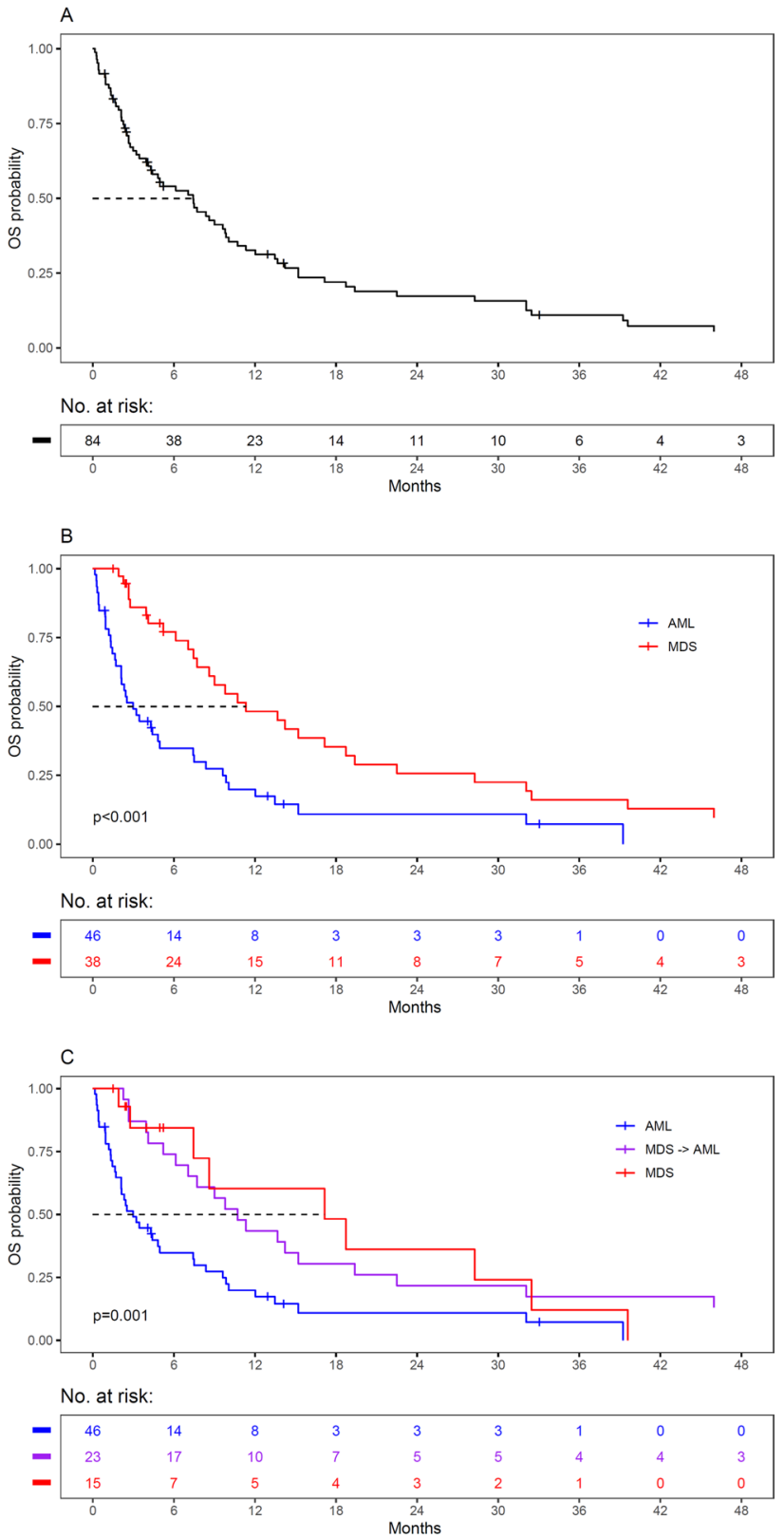

Fig. 4 Survival of patients with AML and MDS with TP53 aberrations. A OS probability of the total cohort of 84 patients. B Survival according to an initial diagnosis of AML or MDS. C Survival of AML, MDS, and transformed MDS patients, calculated from the time of MDS diagnosis

226 days and an estimated 3-year survival rate of $11 \%$, our study confirms previous results reporting an exceedingly adverse outcome of such cohorts [8, 9, 25, 26]. However, in our study, patients with MDS had a significantly better survival than those with AML independently of whether they were treated intensively or non-intensively or received best supportive care. It is well known that in AML and MDS, TP53 mutations are early driver lesions affecting preleukemic/leukemic stem cells. As a sole event, TP53 mutations 
Table 3 Univariable and multivariable Cox regression analyses for overall survival of AML and MDS patients with TP53 aberrations. HR, hazard ratio; $C I$, confidence interval; Ref., reference; $V A F$, variant allele frequency

\begin{tabular}{|c|c|c|c|c|c|}
\hline Variable & Category & $\begin{array}{l}\text { Univariable } \\
\text { HR }(95 \% \mathrm{CI})\end{array}$ & $p$ value & $\begin{array}{l}\text { Multivariable } \\
\text { HR }(95 \% \text { CI })\end{array}$ & $p$ value \\
\hline \multirow[t]{2}{*}{ Group } & AML & 1 (Ref.) & & & \\
\hline & MDS & $0.41(0.25-0.67)$ & $<0.001$ & & \\
\hline \multirow[t]{3}{*}{ Group (3 groups) } & AML & 1 (Ref.) & & & \\
\hline & MDS-> AML & $0.42(0.24-0.73)$ & 0.002 & $0.17(0.07-0.42)$ & $<0.001$ \\
\hline & MDS & $0.39(0.19-0.82)$ & 0.012 & $0.17(0.07-0.41)$ & $<0.001$ \\
\hline Age & & $1.02(0.99-1.04)$ & 0.171 & $1.01(0.97-1.05)$ & 0.499 \\
\hline \multirow[t]{2}{*}{ Sex } & Female & 1 (Ref.) & & 1 (Ref.) & \\
\hline & Male & $0.71(0.44-1.14)$ & 0.154 & $0.75(0.39-1.45)$ & 0.392 \\
\hline VAF & & $1.01(1.00-1.03)$ & 0.026 & $1.02(1.01-1.03)$ & 0.003 \\
\hline \multirow[t]{2}{*}{ Disruptive/Non-disruptive } & Disruptive & 1 (Ref.) & & & \\
\hline & Non-disruptive & $1.68(1.00-2.83)$ & 0.050 & & \\
\hline \multirow[t]{2}{*}{ EAp53 score } & $<75$ & 1 (Ref.) & & & \\
\hline & $\geq 75$ & $0.76(0.44-1.29)$ & 0.310 & & \\
\hline \multirow[t]{2}{*}{ Relative fitness score } & $\leq-0.136$ & 1 (Ref.) & & & \\
\hline & $>-0.136$ & $1.17(0.57-2.39)$ & 0.667 & & \\
\hline \multirow[t]{3}{*}{ Treatment class } & Best supportive care & 1 (Ref.) & & 1 (Ref.) & \\
\hline & Intensive & $0.34(0.18-0.65)$ & 0.001 & $0.08(0.02-0.30)$ & $<0.001$ \\
\hline & Non-intensive & $0.50(0.29-0.88)$ & 0.016 & $0.24(0.10-0.54)$ & 0.001 \\
\hline
\end{tabular}

are unable to induce neoplastic transformation as has been exemplified by their occurrence in healthy individuals with clonal hematopoiesis of indeterminate potential [27]. Secondary events are necessary for full transformation and in both, TP53 mutated AML and MDS, preferably constitute chromosomal aberrations and copy number alterations leading to pronounced expression changes of co-operating gene [28]. Co-operating mutations have also been reported in TP53 aberrant myeloid disorders although their frequency is substantially lower than in AML and MDS with a TP53 wild-type status. In our NGS analysis, co-mutational patterns were different between TP53 aberrant AML and MDS although the number of cases studied was too small to allow for an adequate statistical comparison. Several previous reports have unambiguously determined typical mutational patterns associated with MDS and secondary AML affecting genes like SRSF2 and SF3B1 [29-32]. We, therefore, speculate that the basis for manifestation as either TP53 aberrant AML or MDS as well as their different survival probabilities is mainly due to different co-mutational events occurring secondary to the TP53 aberration. In this regard, it would also be interesting to investigate molecular events responsible for transformation of TP53 aberrant MDS to AML occurring within short periods of time.

The TP53 VAFs were also comparable between AML and MDS. With a median value of $53 \%$ in MDS patients, it indicates that the mutant clone size is considerably exceeding the percentage of bone marrow blast cells in line with previous data on this issue [33]. Interestingly, the TP53 VAF has been shown to be a statistically significant prognostic parameter in this study with higher values having a more pronounced adverse impact on survival. In a study of 1537 AML patients treated intensively within protocols of the AMLSG study group, we were able to demonstrate that even TP53 VAFs of less than $20 \%$ represent significant adverse risk factors for both OS and event-free survival [26]. Furthermore, analysis of the same patient cohort revealed that the specific type of TP53 mutation and its functional consequence has an impact on treatment response [21]. In contrast, in AML patients treated non-intensively with the hypomethylating drug azacitidine, an increasing mutant TP53 load was associated with a significantly increased risk for treatment failure [34]. A systematic review and meta-analysis of studies of patients with MDS also reported a high TP53 VAF as an independent prognostic parameter for survival with a $40 \%$ VAF as cutoff for high and low clonal burdens [35]. Recently, a report focusing on large cohorts of patients with MDS implemented the "TP53 allelic state" as an important biological and clinical parameter. Ideally, the TP53 allelic states should be assessed by combining conventional karyotyping + / FISH and NGS analysis, respectively. Patients with TP53 mono-allelic mutations were comparable to TP53 wild-type patients, whereas multi-hit aberrations consistent with TP53 bi-allelic events showed complex karyotypes and poor outcomes [36]. However, data on the value of the TP53 allelic state in AML are not available yet.

Treatment of patients with TP53 aberrant AML and MDS remains unsatisfactorily; however, novel promising drugs and strategies are being tested in several clinical trials. So far, the only curative approach is allogeneic HSCT 
indicating that restoration of intact immune surveillance mechanisms mediating graft-versus-leukemia takes a central role in combating the disease $[18,37]$. Recently, a phase 3 trial evaluating venetoclax in combination with azacitidine showed superior OS rates in AML patients unfit for intensive therapy. In a subgroup analysis, CR rates for those with TP53 mutations were 55\% treated with that drug combination versus $0 \%$ in the azacitidine group. However, long-term survival did not improve and TP53 mutations could be shown to be a major determinant of resistant disease [38-40]. Evaluating flotetuzumab, a bispecific CD3xCD123 antibody, and magrolimab, a CD47 antibody, together with azacitidine in patients with AML and MDS, respectively, revealed also high remission rates in TP53 aberrant subgroups $[41,42]$. APR-246 (eprenetapopt), a small molecule aiming at shifting mutant p53 towards a wild-type conformation, was recently tested together with azacitidine, in two phase 2 trials in patients with TP53 mutated AML and MDS with encouraging CR and OS rates [43,44]. A consecutive phase 3 study in patients with TP53 mutant MDS failed to meet the primary endpoint of a significantly increased CR rate (Press Releases I Aprea Therapeutics). A comprehensive discussion of ongoing clinical trials in this particular patient cohort has recently been published [45].

In conclusion, our data on TP53 aberrant AML and MDS, obtained at a tertiary cancer center, revealed a high concordance of biological and clinical features between these two disease entities. A significantly increased OS rate in MDS patients is likely due to a different co-mutational pattern. As TP53 aberrations are early leukemogenic events in AML and MDS, these disease entities may, indeed, be classified and treated as a distinct disorder.

Supplementary Information The online version contains supplementary material available at https://doi.org/10.1007/s00277-022-04766-2.

Author contribution HS: conceptualization; SD, GP, GGT, and AW: methodology; GP, GGT, and AB, SD, JM, IB, KL, SR, FL, AR and AZ: data curation; SD, GP, GGT, AW, and HS: original draft preparation; all authors: review, editing, and approval

Funding Open access funding provided by Medical University of Graz. This work was supported by the Austrian Science Fund FWF (grant number P31430-B26) to HS

\section{Declarations}

Ethics approval The study protocol was approved by the ethics committee of the Medical University of Graz, Graz, Austria (vote number 32-644 ex 19/20) and conducted according to the Declaration of Helsinki. Written informed consent was obtained from all patients.
Conflict of interest The authors declare no competing interests.

Open Access This article is licensed under a Creative Commons Attribution 4.0 International License, which permits use, sharing, adaptation, distribution and reproduction in any medium or format, as long as you give appropriate credit to the original author(s) and the source, provide a link to the Creative Commons licence, and indicate if changes were made. The images or other third party material in this article are included in the article's Creative Commons licence, unless indicated otherwise in a credit line to the material. If material is not included in the article's Creative Commons licence and your intended use is not permitted by statutory regulation or exceeds the permitted use, you will need to obtain permission directly from the copyright holder. To view a copy of this licence, visit http://creativecommons.org/licenses/by/4.0/.

\section{References}

1. Kastenhuber ER, Lowe SW (2017) Putting p53 in context. Cell 170:1062-1078

2. Moxley AH, Reisman D (2021) Context is key: understanding the regulation, functional control, and activities of the p53 tumour suppressor. Cell Biochem Funct 39:235-247. https://doi.org/10. 1002/cbf.3590

3. Rivas CI, Wisniewski D, Strife A, Perez A, Lambek C, Bruno S, Darzynkiewicz Z, Clarkson B (1992) Constitutive expression of p53 protein in enriched normal human marrow blast cell populations. Blood 79:1982-1986

4. Liu Y, Elf SE, Miyata Y et al (2009) P53 regulates hematopoietic stem cell quiescence. Cell Stem Cell 4:37-48. https://doi.org/10. 1016/j.stem.2008.11.006

5. Zhao Z, Zuber J, Diaz-Flores E, Lintault L, Kogan SC, Shannon K, Lowe SW (2010) P53 loss promotes acute myeloid leukemia by enabling aberrant self-renewal. Genes Dev 24:1389-1402. https:// doi.org/10.1101/gad.1940710

6. Arber DA, Orazi A, Hasserjian R, Thiele J, Borowitz MJ, Le Beau MM, Bloomfield CD, Cazzola M, Vardiman JW (2016) The 2016 revision to the World Health Organization classification of myeloid neoplasms and acute leukemia. Blood 127:2391-2405. https://doi.org/10.1182/blood-2016-03-643544

7. Reinisch A, Chan SM, Thomas D, Majeti R (2015) Biology and clinical relevance of acute myeloid leukemia stem cells. Semin Hematol 52:150-164. https://doi.org/10.1053/j.seminhematol. 2015.03.008

8. Papaemmanuil E, Gerstung M, Bullinger L et al (2016) Genomic classification and prognosis in acute myeloid leukemia. N Engl J Med 374:2209-2221. https://doi.org/10.1056/NEJMoa1516192

9. Haase D, Stevenson KE, Neuberg D et al (2019) TP53 mutation status divides myelodysplastic syndromes with complex karyotypes into distinct prognostic subgroups. Leukemia 33:17471758. https://doi.org/10.1038/s41375-018-0351-2

10. Woll PS, Kjallquist U, Chowdhury O et al (2014) Myelodysplastic syndromes are propagated by rare and distinct human cancer stem cells in vivo. Cancer Cell 25:794-808. https://doi.org/10.1016/j. ccr.2014.03.036

11. Chen J, Kao YR, Sun D, Todorova TI, Reynolds D, Narayanagari SR, Montagna C, Will B, Verma A, Steidl U (2019) Myelodysplastic syndrome progression to acute myeloid leukemia at the stem cell level. Nat Med 25:103-110. https://doi.org/10.1038/ s41591-018-0267-4 
12. Lal R, Lind K, Heitzer E et al (2017) Somatic TP53 mutations characterize preleukemic stem cells in acute myeloid leukemia. Blood 129:2587-2591. https://doi.org/10.1182/ blood-2016-11-751008

13. Pabst G, Lind K, Graf R, Zebisch A, Stolzel F, Dohner K, Heitzer E, Reinisch A, Sill H (2020) TP53 mutated AML subclones exhibit engraftment in a humanized bone marrow ossicle mouse model. Ann Hematol 99:653-655. https://doi.org/10.1007/ s00277-020-03920-y

14. Schulz E, Valentin A, Ulz P et al (2012) Germline mutations in the DNA damage response genes BRCA1, BRCA2, BARD1 and TP53 in patients with therapy related myeloid neoplasms. J Med Genet 49:422-428. https://doi.org/10.1136/jmedgenet-2011-100674

15. Zebisch A, Lal R, Muller M, Lind K, Kashofer K, Girschikofsky M, Fuchs D, Wolfler A, Geigl JB, Sill H (2016) Acute myeloid leukemia with TP53 germ line mutations. Blood 128:2270-2272

16. Abelson S, Collord G, Ng SWK et al (2018) Prediction of acute myeloid leukaemia risk in healthy individuals. Nature 559:400 404. https://doi.org/10.1038/s41586-018-0317-6

17. Middeke JM, Herold S, Rucker-Braun E et al (2016) TP53 mutation in patients with high-risk acute myeloid leukaemia treated with allogeneic haematopoietic stem cell transplantation. Br J Haematol 172:914-922. https://doi.org/10.1111/bjh.13912

18. Duncavage EJ, Jacoby MA, Chang GS et al (2018) Mutation clearance after transplantation for myelodysplastic syndrome. N Engl J Med 379:1028-1041. https://doi.org/10.1056/NEJMoa1804714

19. Sill H, Zebisch A, Haase D (2020) Acute myeloid leukemia and myelodysplastic syndromes with TP53 aberrations - a distinct stem cell disorder. Clin Cancer Res 26:5304-5309. https://doi. org/10.1158/1078-0432.CCR-20-2272

20. Caraffini V, Geiger O, Rosenberger A et al (2020) Loss of RAF kinase inhibitor protein is involved in myelomonocytic differentiation and aggravates RAS-driven myeloid leukemogenesis. Haematologica 105:375-386. https://doi.org/10.3324/haematol.2018. 209650

21. Dutta S, Pregartner G, Rucker FG, Heitzer E, Zebisch A, Bullinger L, Berghold A, Dohner K, Sill H (2020) Functional classification of TP53 mutations in acute myeloid leukemia. Cancers (Basel) 12:E637. https://doi.org/10.3390/cancers12030637

22. Daga S, Rosenberger A, Kashofer K et al (2020) Sensitive and broadly applicable residual disease detection in acute myeloid leukemia using flow cytometry-based leukemic cell enrichment followed by mutational profiling. Am J Hematol 95:1148-1157. https://doi.org/10.1002/ajh.25918

23. Haubner S, Perna F, Köhnke T et al (2019) Coexpression profile of leukemic stem cell markers for combinatorial targeted therapy in AML. Leukemia 33:64-74. https://doi.org/10.1038/ s41375-018-0180-3

24. Hochberg Y, Benjamini Y (1990) More powerful procedures for multiple significance testing. Stat Med 9:811-818. https://doi.org/ 10.1002/sim.4780090710

25. Bejar R, Stevenson K, Abdel-Wahab O et al (2011) Clinical effect of point mutations in myelodysplastic syndromes. N Engl J Med 364:2496-2506. https://doi.org/10.1056/NEJMoa1013343

26. Prochazka KT, Pregartner G, Rucker FG, Heitzer E, Pabst G, Wolfler A, Zebisch A, Berghold A, Dohner K, Sill H (2019) Clinical implications of subclonal TP53 mutations in acute myeloid leukemia. Haematologica 104:516-523. https://doi.org/10.3324/ haematol.2018.205013

27. Steensma DP, Bejar R, Jaiswal S, Lindsley RC, Sekeres MA, Hasserjian RP, Ebert BL (2015) Clonal hematopoiesis of indeterminate potential and its distinction from myelodysplastic syndromes. Blood 126:9-16. https://doi.org/10.1182/ blood-2015-03-631747

28. Rucker FG, Dolnik A, Blatte TJ et al (2018) Chromothripsis is linked to TP53 alteration, cell cycle impairment, and dismal outcome in acute myeloid leukemia with complex karyotype. Haematologica 103:e17-e20. https://doi.org/10.3324/haematol.2017. 180497

29. Lindsley RC, Mar BG, Mazzola E et al (2015) Acute myeloid leukemia ontogeny is defined by distinct somatic mutations. Blood 125:1367-1376. https://doi.org/10.1182/blood-2014-11-610543

30. Dohner H, Weisdorf DJ, Bloomfield CD (2015) Acute myeloid leukemia. N Engl J Med 373:1136-1152. https://doi.org/10.1056/ NEJMra1406184

31. Sperling AS, Gibson CJ, Ebert BL (2017) The genetics of myelodysplastic syndrome: from clonal haematopoiesis to secondary leukaemia. Nat Rev Cancer 17:5-19. https://doi.org/10.1038/nrc. 2016.112

$32 \mathrm{Yu}$ J, Li Y, Li T et al (2020) Gene mutational analysis by NGS and its clinical significance in patients with myelodysplastic syndrome and acute myeloid leukemia. Exp Hematol Oncol 9:2-5. eCollection 2020. https://doi.org/10.1186/s40164-019-0158-5

33. Walter MJ, Shen D, Ding L et al (2012) Clonal architecture of secondary acute myeloid leukemia. N Engl J Med 366:1090-1098. https://doi.org/10.1056/NEJMoa1106968

34. Dohner H, Dolnik A, Tang L et al (2018) Cytogenetics and gene mutations influence survival in older patients with acute myeloid leukemia treated with azacitidine or conventional care. Leukemia 32:2546-2557. https://doi.org/10.1038/s41375-018-0257-Z

35. Deng J, Wu X, Ling Y, Liu X, Zheng X, Ye W, Gong Y (2020) The prognostic impact of variant allele frequency (VAF) in TP53 mutant patients with MDS: a systematic review and meta-analysis. Eur J Haematol 105:524-539. https://doi.org/10.1111/ejh.13483

36. Bernard E, Nannya Y, Hasserjian RP et al (2020) Implications ofTP53allelic state for genome stability, clinical presentation and outcomes in myelodysplastic syndromes. Nat Med 26:1549-+. https://doi.org/10.1038/s41591-020-1008-z

37. Ciurea SO, Chilkulwar A, Saliba RM et al (2018) Prognostic factors influencing survival after allogeneic transplantation for AML/MDS patients with TP53 mutations. Blood 131:2989-2992. https://doi.org/10.1182/blood-2018-02-832360

38. DiNardo CD, Jonas BA, Pullarkat V et al (2020) Azacitidine and venetoclax in previously untreated acute myeloid leukemia. $\mathrm{N}$ Engl J Med 383:617-629. https://doi.org/10.1056/NEJMoa2012 971

39. DiNardo CD, Tiong IS, Quaglieri A et al (2020) Molecular patterns of response and treatment failure after frontline venetoclax combinations in older patients with AML. Blood 135:791-803. https://doi.org/10.1182/blood.2019003988

40. Pollyea DA, Pratz KW, Wei AH et al (2021) Outcomes in patients with poor-risk cytogenetics with or without TP53 mutations treated with venetoclax combined with hypomethylating agents. Blood 138(Supplement 1):224

41. Vadakekolathu J, Lai C, Reeder S et al (2020) TP53 abnormalities correlate with immune infiltration and associate with response to flotetuzumab immunotherapy in AML. Blood Adv 4:5011-5024. https://doi.org/10.1182/bloodadvances.2020002512

42. Chao MP, Takimoto CH, Feng DD, McKenna K, Gip P, Liu J, Volkmer JP, Weissman IL, Majeti R (2020) Therapeutic targeting of the macrophage immune checkpoint CD47 in myeloid malignancies. Front Oncol 9:1380. https://doi.org/10.3389/fonc.2019. 01380 
43. Sallman DA, DeZern AE, Garcia-Manero G et al (2021) Eprenetapopt (APR-246) and azacitidine in TP53-mutant myelodysplastic syndromes. J Clin Oncol 39:1584-1594. https://doi.org/10.1200/ JCO.20.02341

44. Cluzeau T, Sebert M, Rahmé R et al (2021) Eprenetapopt plus azacitidine in TP53-mutated myelodysplastic syndromes and acute myeloid leukemia: a phase II study by the Groupe Francophone des Myélodysplasies (GFM). J Clin Oncol 39:1575-1583. https://doi.org/10.1200/JCO.20.02342
45. Jiang Y, Gao SJ, Soubise B, Douet-Guilbert N, Liu ZL, Troadec MB (2021) TP53 in myelodysplastic syndromes. Cancers (Basel) 13:5392. https://doi.org/10.3390/cancers 13215392

Publisher's note Springer Nature remains neutral with regard to jurisdictional claims in published maps and institutional affiliations. 